\title{
Toward Identifying the QCD Critical Point: attenuation of the sound mode around the critical point
}

\author{
Teiji Kunihiro* and Yuki Minami \\ Department of Physics, Kyoto University, Kyoto 606-8502, Japan \\ E-mail: kunihiro@ruby.scphys.kyoto-u.ac.jp, \\ y-minamieruby.scphys.kyoto-u.ac.jp
}

\begin{abstract}
Motivated by the fact that QCD critical point (CP) belongs to the same universality class as the liquid-gas transition, the dynamical density fluctuations around the $\mathrm{CP}$ is analyzed using relativistic fluid dynamics for a viscous fluid. It is shown that relativistic effects on the dynamical structure factor of the density fluctuation appears only in the width of the sound and thermal modes through a modification of the transport/kinetic coefficients. It is found that the mechanical density fluctuation which is enhanced by the relativistic effects are attenuated, whereas the entropy fluctuation in turn becomes more prominent around the QCD CP. This attenuation of the density fluctuation around the QCD CP may imply that if the suppression or even total disappearance of Mach cone is observed as the incident energy of the relativistic heavy-ion collisions is lowered, it can be a signal of the existence of the QCD critical point.
\end{abstract}

5th International Workshop on Critical Point and Onset of Deconfinement - CPOD 2009,

June 08 - 122009

Brookhaven National Laboratory, Long Island, New York, USA

\footnotetext{
* Speaker.
} 


\section{Introduction}

An interesting feature of the QCD phase diagram is the existence of a critical point[1, 2]; see Refs. [3, 4, 5] for possible variants and alternatives. At the QCD critical point (CP), the first order phase transition terminates and turns to a second order phase transition. It is now known that the QCD CP belongs to the universality class $\mathrm{H}$ in the classification scheme by HohengergHalperin[[]], i.e., the same as that of the liquid-gas phase transition point[[], \&]. This implies that the density fluctuating mode and, generically, fluid dynamic modes coupled to conserved quantities are the softening modes at the CP.

The $\sigma$ mode that is coupled to the fluctuation of the chiral order parameter $\langle\bar{q} q)\rangle$ becomes the soft mode of the chiral transition at finite temperature $T$ but vanishing chemical potential $\mu=0$. The QCD CP exists, however, at finite chemical potential $\mu$ and for finite current quark masses. In such a case, as is first shown in [9], the scalar-vector cross term $\left\langle:\left(\bar{q} \gamma^{0} q\right)(\bar{q} q):\right\rangle$ does not vanish, and, hence, the scalar mode is coupled with the density-density correlator $\left\langle:\left(\bar{q} \gamma^{0} q\right)^{2}:\right\rangle$; this is because charge conjugation symmetry is violated with finite $\mu$, and the left- and right-handed quarks are coupled owing to the breaking of chiral symmetry. ${ }^{1}$ Then, the would-be soft mode, the $\sigma$ mode, remains massive (a fast mode) and becomes a slaving mode of the genuine soft mode given by the fluid dynamical modes coupled to the density fluctuation[7], 8]. Here we note that the diverging behavior of the density fluctuation around the QCD CP was first shown in [10]; see also [11].

Recently, it has been argued [12] on the basis of the analysis of the liquid-gas phase transition by Onuki[13] that the bulk viscosity may show a singular behavior around the QCD CP.

We explore how the singularities of the thermodynamic quantities as well as the transport coefficients affect the dynamical density fluctuations around the QCD CP thereby we can have a hint how to identify the signal of the existence of QCD CP by experiment[14]. Our investigation is based on an explicit use of the relativistic fluid dynamic equations for a viscous fluid. We also demonstrate that even the so called first-order relativistic fluid dynamic equations have generically no problem to describe fluid dynamical phenomena with long wave lengths contrary to a possible naive suspect.

We find that the mechanical density fluctuation which is enhanced by the relativistic effects are attenuated, but the entropy fluctuation in turn becomes more prominent around the QCD CP, which is, to our surprise, precisely the same in effect as the critical behavior in the nonrelativistic case. [15] On the basis of these findings, we speculate that if a Mach cone, which originates from the dynamical density fluctuations stimulated by a fast particle and seems to have been observed in RHIC experiment[16], disappears or is strongly suppressed as the incident energy of relativistic heavy-ion collisions is lowered, it can be a signal of the existence of the QCD CP provided that the incident energy is still high enough to make jets[17].

\footnotetext{
${ }^{1}$ It is also notable that the density-density correlator is a part of the polarization tensor $\left\langle:\left(\bar{q} \gamma^{\mu} q\right)\left(\bar{q} \gamma^{\nu} q\right):\right\rangle$, which suggests that the vector coupling $g_{V}\left(\bar{q} \gamma^{\mu} q\right)^{2}$ plays an important role for the static and dynamic properties of the QCD CP.[9]
} 


\section{Dissipative Relativistic Fluid Dynamics}

Relativistic fluid dynamic equations consist of the balance equations for energy-momentum and particle number, $\partial_{\mu} T^{\mu v}=0$ and $\partial_{\mu} N^{\mu}=0$ where $T^{\mu v}$ is the energy-momentum tensor and $N^{\mu}$ the particle current:

$$
T^{\mu v}=(\varepsilon+P) u^{\mu} u^{v}-P g^{\mu v}+\tau^{\mu v}, \quad N^{\mu}=n u^{\mu}+v^{\mu} .
$$

Here $\varepsilon$ is the energy density, $P$ the pressure, $u^{\mu}$ the flow velocity, and $n$ the particle density; the dissipative part of the energy-momentum tensor and the particle current are denoted by $\tau^{\mu v}$ and $v^{\mu}$, respectively.

The so called first-order equations such as Landau[18] and Eckart[19] ones are parabolic and formally violates the causality, and, hence, are called acausal. Moreover, the Eckart equation which is defined for the particle frame where the particle current does not have a dissipative part $\left(v^{\mu}=0\right)$ shows a pathological property that the fluctuations around the thermal equilibrium is unstable[20]. The causality problem is circumvented in the Israel-Stewart equation[21], which is a second-order equation with relaxation times incorporated.

One should, however, notice that formally acausal fluid dynamic equations should be valid in describing fluid dynamical phenomena with longer wavelengths than the mean free path. In fact, we will see that the results for fluid dynamical modes with long wave lengths are qualitatively the same irrespective whether the second-order or first-order equations are used.

As for the instability seen in the Eckart equation, a new first-order equation in the particle frame constructed by Tsumura, Kunihiro and Ohnishi (TKO) [22] has no such a pathological behavior[23]. We employ Landau[18], Eckart[19] and Israel-Stewart(I-S)[21] equation as typical equations, and TKO equation in particle frame.

\section{Dynamic structure factor of density fluctuations}

In this section, we first present a procedure for deriving the dynamical structure factor (spectral function) of the density fluctuation for the Landau equation. Then we just give the results for Eckart, Tsumura-Kunihiro-Ohnishi(TKO) equation and the Israel-Stewart equation in particle frame.

\subsection{Landau equation (energy frame)}

The dissipative terms in the Landau equation are given by

$$
\tau^{\mu v}=\eta\left[\partial_{\perp}^{\mu} u^{v}+\partial_{\perp}^{v} u^{\mu}-\frac{2}{3} \Delta^{\mu v}\left(\partial_{\perp} \cdot u\right)\right]+\zeta \Delta^{\mu v}\left(\partial_{\perp} \cdot u\right), \quad v^{\mu}=\kappa\left(\frac{n T}{w}\right)^{2} \partial_{\perp}^{\mu}\left(\frac{\mu}{T}\right),
$$

where $\eta$ is the shear viscosity, $\zeta$ the bulk viscosity, $\kappa$ the thermal conductivity and $w=\varepsilon+P$ the enthalpy density. $\Delta^{\mu v}=g^{\mu v}-u^{\mu} u^{v}$ is the projection operator on the space-like vector, and $\partial_{\perp}^{\mu}=\Delta^{\mu v} \partial_{v}$ the space-like derivative (gradient operator).

We calculate the dynamical structure factor, or the spectral function, of the density fluctuation around the thermal equilibrium state, as was done for the non-relativistic case[24, 25]; we refer to the comprehensive text book Ref. [25]. 
Let us express the dynamical quantities as follows, $n(x)=n_{0}+\delta n(x), \varepsilon(x)=\varepsilon_{0}+\delta \varepsilon(x)$, $P(x)=P_{0}+\delta P(x), \mu(x)=\mu_{0}+\delta \mu(x)$, and $u^{\mu}(x)=u_{0}^{\mu}+\delta u^{\mu}(x)$, where the variables with the subscript ${ }_{0}$ denote those in the equilibrium state in at rest; $u_{0}^{\mu}=(1, \mathbf{0})$. Then, $\delta u^{\mu}(x)=(0, \delta \mathbf{v}(x))$ with $\delta \mathbf{v}(x)$ yet to be determined together with other quantities like $\delta n$ etc. Now we have five equations for seven unknown quantities, $\delta n, \delta T, \delta P, \delta s$, and $\delta \mathbf{v}$. Choosing $\delta n$ and $\delta T$ as independent variables, we have $\delta P(x)=\frac{w_{0} c_{s}^{2}}{n_{0} \gamma} \delta n(x)+\frac{w_{0} c_{s}^{2} \alpha_{P}}{\gamma} \delta T(x)$ and $\delta s(x)=-\frac{w_{0} c_{s}^{2} \alpha_{P}}{n_{0}^{2} \gamma} \delta n(x)+\frac{\tilde{c}_{n}}{T_{0}} \delta T(x)$. Here $\tilde{c}_{n}=T_{0}(\partial s / \partial T)_{n}$ and $\tilde{c}_{P}=T_{0}(\partial s / \partial T)_{P}$ are the specific heats at constant density and pressure, respectively, $c_{s}=(\partial P / \partial \varepsilon)_{s}^{1 / 2}$ the sound velocity, $\alpha_{P}=-\left(1 / n_{0}\right)(\partial n / \partial T)_{P}$ the thermal expansivity at constant pressure, and $\gamma=\tilde{c}_{P} / \tilde{c}_{n}$ the ratio of the specific heats. The equation describing the density fluctuations is finally found to be

$$
\begin{array}{r}
\left\{\frac{\partial}{\partial t}-\kappa \frac{T_{0} c_{s}^{2}}{w_{0} \gamma} \nabla^{2}\right\} \delta n+n_{0} \nabla \cdot \delta \mathbf{v}+\kappa \frac{n_{0}}{w_{0}}\left(1-\frac{c_{s}^{2} \alpha_{P} T_{0}}{\gamma}\right) \nabla^{2} \delta T=0, \\
w_{0} \frac{\partial \delta \mathbf{v}}{\partial t}-\eta \nabla^{2} \delta \mathbf{v}-\left(\zeta+\frac{1}{3} \eta\right) \nabla(\nabla \cdot \delta \mathbf{v})+\frac{w_{0} c_{s}^{2}}{n_{0} \gamma} \nabla \delta n+\frac{w_{0} c_{s}^{2} \alpha_{P}}{\gamma} \nabla \delta T=0, \\
\left\{-\frac{w_{0} c_{s}^{2} \alpha_{P}}{n_{0} \gamma} \frac{\partial}{\partial t}+\kappa \frac{c_{s}^{2}}{n_{0} \gamma} \nabla^{2}\right\} \delta n+\left\{\frac{n_{0} \tilde{c}_{n}}{T_{0}} \frac{\partial}{\partial t}+\kappa\left(\frac{c_{s}^{2} \alpha_{P}}{\gamma}-\frac{1}{T_{0}}\right) \nabla^{2}\right\} \delta T=0 .
\end{array}
$$

Now we are interested in the dynamical structure factor of the density fluctuation as given by

$$
S_{n n}(\mathbf{k}, \omega) \equiv\langle\delta \tilde{n}(\mathbf{k}, \omega) \delta n(\mathbf{k}, t=0)\rangle,
$$

where $\delta \tilde{n}(\mathbf{k}, \omega)$ is the Fourier transform of the density fluctuation, and \langle\rangle denotes the thermal average in the equilibrium.

Note that $\delta T$ and $\delta n$ are statistically independent in fluid systems which is established in Einstein fluctuation theory[25]; $\langle\delta T(\mathbf{k}, 0) \delta n(\mathbf{k}, 0)\rangle=0$, and similarly, $\left\langle\delta v_{\|}(\mathbf{k}, 0) \delta n(\mathbf{k}, 0)\right\rangle=0$, where $\delta v_{\|}(\mathbf{k}, 0)$ denotes the longitudinal component of the velocity field. Thus we have the dynamical structure factor

$$
\frac{S_{n n}(\mathbf{k}, \omega)}{\left\langle(\delta n(\mathbf{k}, t=0))^{2}\right\rangle}=\left(1-\frac{1}{\gamma}\right) \frac{2 \Gamma_{\mathrm{R}} k^{2}}{\omega^{2}+\Gamma_{\mathrm{R}}^{2} k^{4}}+\frac{1}{\gamma}\left\{\frac{\Gamma_{\mathrm{B}} k^{2}}{\left(\omega-c_{s} k\right)^{2}+\Gamma_{\mathrm{B}}^{2} k^{4}}+\frac{\Gamma_{\mathrm{B}} k^{2}}{\left(\omega+c_{s} k\right)^{2}+\Gamma_{\mathrm{B}}^{2} k^{4}}\right\} .
$$

Here, $\Gamma_{\mathrm{R}}=\kappa /\left(n_{0} \tilde{c}_{P}\right)$ and $\Gamma_{\mathrm{B}}=\frac{1}{2}\left[\chi(\gamma-1)+v_{l}\right]+\frac{1}{2} c_{s}^{2} T_{0}\left(\frac{\kappa}{w_{0}}-2 \chi \alpha_{P}\right)$ with $v_{l}=(\zeta+4 \eta / 3) / w_{0}$ being the (relativistic) longitudinal kinetic viscosity; notice $w_{0}$ in the denominator. We also remark that the width $\Gamma_{\mathrm{R}}$ is identified with the thermal diffusivity $\chi$.

We see that the spectral function has three peaks at frequencies $\omega=0$ and $\omega= \pm c_{s} k$ : The peak at $\omega=0$ corresponds to thermally induced density fluctuations, which is called Rayleigh peak; while the two-side peaks at $\omega= \pm c_{s} k$ correspond to mechanically induced density fluctuation, i.e. sound waves. These two peaks are called Brillioun peaks.

We find that relativistic effects manifest themselves only in the width of the Brillouin peaks $\Gamma_{\mathrm{B}}$, whereas the width of the Rayleigh peak is the same as the non-relativistic case [24, 25]. The relativistic effects in $\Gamma_{\mathrm{B}}$ appear in two ways, as seen by the expression,

$$
\Gamma_{\mathrm{B}}=\Gamma_{\mathrm{B}}^{\mathrm{MR}}+\delta \Gamma_{\mathrm{B}}^{\mathrm{La}}
$$

where $\Gamma_{\mathrm{B}}^{\mathrm{MR}} \equiv \frac{1}{2}\left[\chi(\gamma-1)+v_{l}\right]$ and $\delta \Gamma_{\mathrm{B}}^{\mathrm{La}} \equiv \frac{1}{2} c_{s}^{2} T_{0}\left(\kappa / w_{0}-2 \chi \alpha_{P}\right)$. Firstly, the first term has a nonrelativistic counter part only with a replacement of the enthalpy density $w_{0}$ with the mass density $\rho_{0}$ 

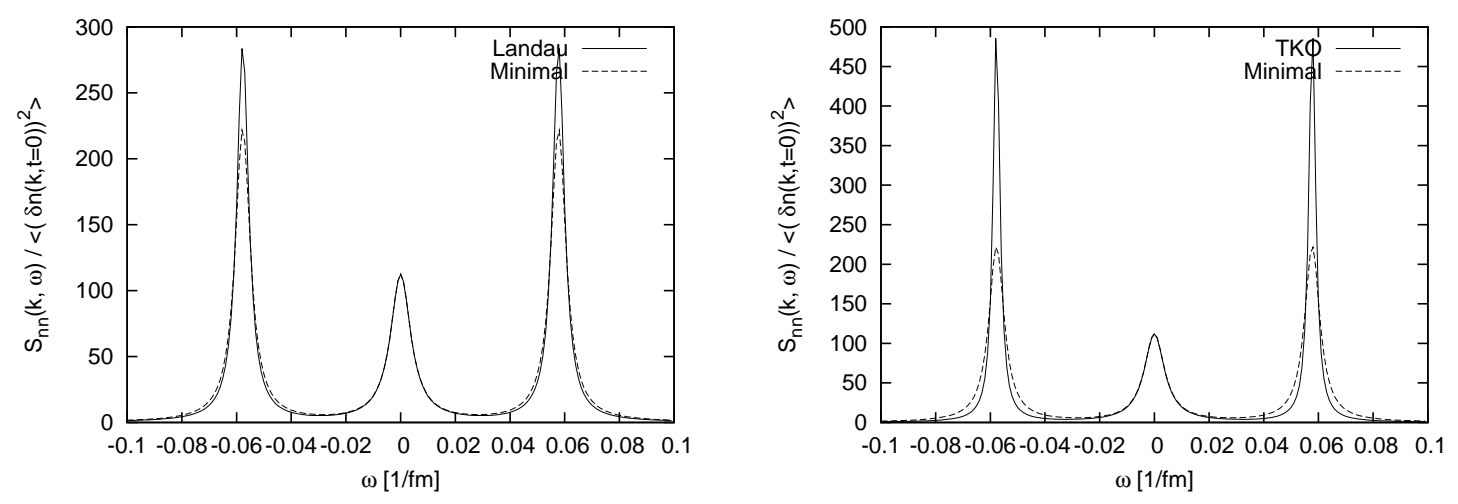

Figure 1: Left panel: The solid line is the spectral function for the density fluctuations from Landau/IsraelStewart equation with $k=0.1[1 / \mathrm{fm}], \mu_{0}=200[\mathrm{MeV}], T_{0}=200[\mathrm{MeV}], \eta /\left(n_{0} s_{0}\right)=\zeta /\left(n_{0} s_{0}\right)=0.3$ and $\kappa T_{0} /\left(n_{0} s_{0}\right)=0.6$; the dashed line in the minimal relativistic case. Right panel: the result from TKO equation with the same parameters. Notice that the vertical scale is different from that of the left panel.

in $v_{l}$. We call this modification the minimal relativistic (MR) effect. On the other hand, the second term $\delta \Gamma_{B}^{\mathrm{La}}$ is a genuine relativistic effect that is absent in the non-relativistic case; this comes from the mass-energy equivalence inherent in the relativistic theory.

Figure 11 shows the spectral function Eq. (3.6) and the minimal relativistic case with $\delta \Gamma_{B}=0$ for the parameter set $k=0.1[1 / \mathrm{fm}], \mu_{0}=200[\mathrm{MeV}], T_{0}=200[\mathrm{MeV}], \eta /\left(n_{0} s_{0}\right)=\zeta /\left(n_{0} s_{0}\right)=0.3$ and $\kappa T_{0} /\left(n_{0} s_{0}\right)=0.6$. Note that $n_{0} s_{0}$ represents the entropy density in the equilibrium state because $s_{0}$ is the entropy per particle number. As is expected, Fig 1 shows that the Brillouin peaks owing to the sound mode is enhanced by the relativistic effects, while the Rayleigh peak owing to the thermal mode is the same as in the non-relativistic case.

\subsection{Stable first-order fluid dynamic equation in the particle frame}

In the case of TKO equation[22] in the particle frame $\left(v^{\mu}=0\right)$, the dissipative terms are given by

$$
\tau^{\mu v}=\eta\left[\partial_{\perp}^{\mu} u^{v}+\partial_{\perp}^{v} u^{\mu}-\frac{2}{3} \Delta^{\mu v}\left(\partial_{\perp} \cdot u\right)\right]-\zeta^{\prime}\left(3 u^{\mu} u^{v}-\Delta^{\mu v}\right)\left(\partial_{\perp} \cdot u\right)+\kappa\left(u^{\mu} \partial_{\perp}^{v} T+u^{v} \partial_{\perp}^{\mu}\right)
$$

where $\zeta^{\prime}=\zeta /(3 \gamma-4)^{2}$. From the same procedure as taken for the Landau equation, we obtain the dynamical structure factor with the same form as (3.6) but with a different form of the width of the Brillouin peaks[14];

$$
\Gamma_{\mathrm{B}}=\frac{1}{2}\left[\chi(\gamma-1)+v_{l}^{\mathrm{TKO}}-\frac{\alpha_{P} c_{s}^{2}}{n_{0} \tilde{c}_{P}}\left(\kappa T_{0}+3 \zeta^{\prime}\right)\right] \equiv \Gamma_{\mathrm{B}}^{\mathrm{TKO}} .
$$

We emphasize that since the fluid dynamic fluctuations around the equilibrium state is relaxing in TKO equation, we have obtained the dynamical structure factor without any obstruction, in contrast to the case of Eckart equation[19], for which we have found[14] that the unstable behavior of the density fluctuation around the thermal equilibrium prevents us from obtaining the dynamical structure factor in a sensible way. 
We see that the genuine relativistic effect in the width of the Brillouin peaks reads $\delta \Gamma_{\mathrm{B}}^{\mathrm{TKO}} \equiv$ $-\frac{\alpha_{P} c_{s}^{2}}{2 n_{0} \tilde{c}_{P}}\left(\kappa T_{0}+3 \zeta^{\prime}\right)$, which is definitely negative, implying that the relativistic effect acts to enhance and sharpen the spectral function of the density fluctuation in comparison with the minimal relativistic case than in the energy frame; see the right panel in Fig.1; notice that the scales of the right and left panel are different.

\subsection{Israel-Stewart equation}

The derivation of the dynamical structure factor for the Israel-Stewart (I-S) equation[21] can be performed much the same way as for the Landau equation with some additional complications due to the presence of the relaxation times. However, it is found that if the relaxation time $\beta_{1}$ of the heat current is so small as to satisfy the condition $\beta_{1}<\frac{1}{w_{0}}$, the I-S equation in particle frame takes over the pathological behavior of Eckart one even though the relaxation time is finite; i.e., the density fluctuation will not relax down.

If we assume that the relaxation time is sufficiently large to satisfy the inequality $\beta_{1}>\frac{1}{w_{0}}$, then we can have the spectral function,

$$
\begin{aligned}
S_{n n}(\mathbf{k}, \omega) /\left\langle(\delta n(\mathbf{k}, t=0))^{2}\right\rangle= & \left(1-\frac{1}{\gamma}\right) \frac{2 \chi k^{2}}{\omega^{2}+\chi^{2} k^{4}}+\frac{1}{\gamma}\left[\frac{\Gamma_{\mathrm{B}} k^{2}}{\left(\omega-c_{s} k\right)^{2}+\Gamma_{\mathrm{B}}^{2} k^{4}}\right. \\
& \left.+\frac{\Gamma_{\mathrm{B}} k^{2}}{\left(\omega+c_{s} k\right)^{2}+\Gamma_{\mathrm{B}}^{2} k^{4}}\right]+O\left(k^{2}\right) \times\left[\frac{2 / \beta_{0} \zeta}{\omega^{2}+1 /\left(\beta_{0} \zeta\right)^{2}}\right. \\
& \left.+\frac{1 / \beta_{2} \eta}{\omega^{2}+1 /\left(2 \beta_{2} \eta\right)^{2}}+\frac{2 w_{0} /\left[\left(\beta_{1} w_{0}-1\right) \kappa T_{0}\right]}{\omega^{2}+w_{0}^{2} /\left[\left(\beta_{1} w_{0}-1\right) \kappa T_{0}\right]^{2}}\right] .
\end{aligned}
$$

Here, $\beta_{0}$ and $\beta_{2}$ denote the relaxation times for the bulk viscosity and the shear viscosity, respectively. Apparently the spectral function has six peaks including the conventional three peaks, but the new three Lorentzian functions should vanish in the long wavelength limit $k \rightarrow 0$, because the strength of these is in the second order of $k$. Therefore Israel-Stewart equation gives completely the same result for the dynamical structure factor for the density fluctuations in the long wavelength limit as that Landau equation gives. That is, the relaxation times do not affect the result in the fluid dynamical regime.

\section{The behavior around the QCD critical point}

We are now in a position to analyze the behavior of the spectral function of the density fluctuations around the QCD critical point, on the basis of the dynamic as well as static scaling laws for the liquid-gas transition [15, 13].

The specific heat at constant density $\tilde{c}_{n}$ and the the isothermal compressibility $K_{T}=\frac{1}{n_{0}}\left(\frac{\partial n}{\partial P}\right)_{T}$ diverge at the critical point as $\tilde{c}_{n}=c_{0} t^{-\tilde{\alpha}}$ and $K_{T}=K_{0} t^{-\tilde{\gamma}}$, where $t=\left|\left(T-T_{c}\right) / T_{c}\right|$ is a reduced temperature. The values of these critical exponents are known to be $\tilde{\alpha} \sim 0.11$ and $\tilde{\gamma} \sim 1.2$, respectively.

At the critical point, the correlation length $\xi$ diverges as $\xi=\xi_{0} t^{-v}$ with $v \sim 0.63$. The bulk viscosity may also show a singular behavior around the QCD CP, as shown in [13, 12]; $\zeta=\zeta_{0} t^{-a_{\zeta}}$ with $\zeta_{0}$ being a constant. The exponent $a_{\zeta}$ for the liquid-gas transition is predicted [13] to be $z v-\tilde{\alpha}$ 
with the dynamical critical exponent $z ; z \sim 3$. Thus we have $a_{\zeta} \sim 1.8$. The singular behavior of the thermal conductivity around the critical point is given by $a_{\kappa} ; \kappa=\kappa_{0} t^{-a_{\kappa}}$ with $a_{\kappa} \sim 0.63$ where $\kappa_{0}$ is a constant.

Now using these formulae, we can show[14] that the width of the Rayleigh peak behaves as $\Gamma_{\mathrm{R}} \sim t^{\tilde{\gamma}-a_{\kappa}}$, which tells us that the width $\Gamma_{\mathrm{R}}$ becomes narrow as the QCD CP is approached. We emphasize that this result is independent of the choice of the relativistic fluid dynamic equation or frame.

We find that the width of the Brillouin peaks $\Gamma_{\mathrm{B}}$ show the following critical behavior for the Landau and I-S equations, $\Gamma_{\mathrm{B}} \sim \frac{\zeta_{0}}{2 w_{0}} t^{-a_{\zeta}}$. We note that this singularity comes from that of the bulk viscosity. In the case of TKO equation, we first note that the critical behavior of the effective bulk viscosity is given as $\zeta^{\prime}=\frac{\zeta}{(3 \gamma-4)^{2}} \sim t^{2 \tilde{\gamma}-a_{\zeta}}$, which shows that the effective bulk viscosity has a positive exponent and does not show a singular behavior because $a_{\zeta} \sim 1.8$ and $\tilde{\gamma} \sim 1.2$. Instead, the singularity of the Brillouin peaks for TKO equation comes from that of the thermal conductivity[14]; $\Gamma_{\mathrm{B}} \sim t^{-\left(a_{\kappa}-\tilde{\alpha}\right)}$.

Anyway, we have found that the width $\Gamma_{\mathrm{B}}$ diverges at the QCD CP, irrespective of the relativistic fluid dynamic equations, although the strength of the singularity may differ depending on the choice of the fluid dynamic equation[14].
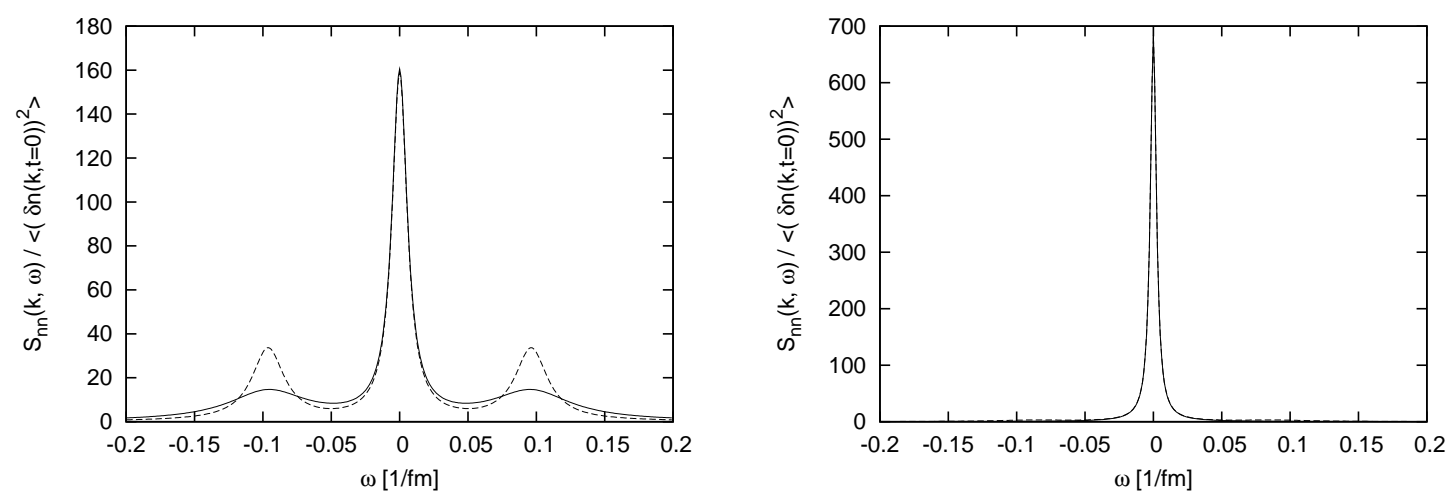

Figure 2: Left panel: The spectral function at $t \equiv\left|T-T_{c}\right| / T_{C}=0.4$ and $k=0.1[1 / \mathrm{fm}]$. The solid line represents the Landau and I-S cases. The dashed line represents the TKO case. The strength of the Brillouin peaks becomes small due to the singularity of the ratio of specific heats. Right panel:The spectral function at $t=0.1$ and $k=0.1[1 / \mathrm{fm}]$. We see that the Brillouin peaks which correspond to sound wave dies out and the difference between the Landau and TKO cases disappears.

It turns out that these singular behaviors of the width of the Brillouin peaks around the QCD $\mathrm{CP}$ may not be observed: Note that the strengths of the Rayleigh and the Brillouin peaks are given in terms of $\gamma$, which behaves like $\gamma=\tilde{c}_{p} / \tilde{c}_{n} \sim t^{-\tilde{\gamma}+\tilde{\alpha}} \rightarrow \infty$, in the critical region. Then the strength of the Brillouin peaks is attenuated and only the Rayleigh peak stands out in the critical region, as follows;

$$
S_{n n}(\mathbf{k}, \omega) \sim\left\langle(\delta n(\mathbf{k}, 0))^{2}\right\rangle \frac{2 \Gamma_{\mathrm{R}} k^{2}}{\omega^{2}+\Gamma_{\mathrm{R}}^{2} k^{4}}, \quad\left(T \sim T_{c}\right) .
$$

Figure 2 shows how the dynamical structure factor for the density fluctuations in the Landau (I$\mathrm{S})$ and TKO cases behaves around the QCD critical point for $k=0.1[1 / \mathrm{fm}], t=0.5$ and $t=0.1$, 
respectively. We see that the strength of the Brillouin peaks becomes small and dies out as the system approach the QCD CP. In addition, the static correlation function $\left\langle(\delta n(\mathbf{k}, t=0))^{2}\right\rangle$ shows a singular behavior in the forward direction $k=0$. This is known as the critical opalescence[15, 25]. Then the strength of the Rayleigh peak will be most drastically enhanced in the forward angle in the critical region.

Why at all do sound modes or mechanical density fluctuations die out at the critical point? To answer this question, we first note that the fluid dynamic regime is expressed as $\xi<<\lambda_{s}$, where $\lambda_{s}$ represents the wave length of the sound mode. When this condition is satisfied, the sound mode can be developed[6]. However, in the vicinity of the critical point, the correlation length $\xi$ becomes very large and eventually goes to infinity, so the above inequality can not be satisfied, and the sound mode is hardly developed in the vicinity of the critical point[6]. This is the reason why the sound mode or density fluctuation is hardly developed around the QCD critical point.

\section{Possible disappearance or suppression of Mach cone around the QCD CP}

The result in the last section suggests that phenomena inherently related to the existence of the mechanical density fluctuations may disappear around the critical point. One of such phenomena is the possible Mach cone formation [17] by the particle passing through the medium with a speed larger than the sound velocity $c_{s}$. Such a Mach-cone like particle correlations are observed in the RHIC experiment[16]. If such three-particle correlations have been confirmed to be a Mach-cone formation, then the disappearance or suppression of the Mach cone would be a signal that the created matter has passed through the critical region, showing the existence of the QCD critical point. Even if the thermal wake also contributes to the formation of Mach cone, a suppression of Mach cone may be expected by the attenuation of the dynamical density fluctuations. So it would be very interesting to see possible variation of the strength of the Mach cone according to the variation of the incident energy of the heavy-ion collisions. In theoretical side, it is an intriguing task to explore the fate of Mach cone with an equation of state that admits the existence of the CP.

\section{Summary and concluding remarks}

Motivated by the fact that QCD critical point (CP) belongs to the same universality class as the liquid-gas transition, we have analyzed the dynamical density fluctuations using relativistic fluid dynamics. We have found that the mechanical density fluctuations are attenuated owing to the divergence of the correlation length $\xi$ around the QCD CP; on the other hand, the entropy fluctuation in turn gets enhanced and tends to makes a single peak around the QCD CP in the dynamical structure factor of the density fluctuations. Sunch an attenuation of the mechanical density fluctuations may lead to a suppression or even total disappearance of Mach cone at the QCD CP. If the Mach cone formation is confirmed at the incident energy $\sqrt{s_{N N}}=200 \mathrm{GeV}$ in RHIC experiments, possible disappearance or strong suppression of a Mach cone along with the lowering of the incident energy can be a signal of the existence of the critical point; the created matter should have gone through the critical region of the CP. 
Eexplicit calculations with equation of motion which admittes the existence of the critical point is necessary for confirm the fate of Mach cone formation. To make a direct connection with RHIC experiment, we should analyze the density fluctuations with the expanding back ground[26].

As a future work, we should study the coupling between the thermal fluctuations and the transverse mode using the mode-mode coupling theory in the close vicinity of the critical point for the relativistic case.

Finally, we would like to indicate that there are still other possibilities in the structure of the QCD phase diagram [3, 4, 5]: For example, there may exist multiple critical points when the color superconductivity and the vector interaction like $g_{V}\left(\bar{q} \gamma^{\mu} q\right)^{2}[$ [3] and/or the anomaly term[ [4 are incorporated. It suggests that the QCD matter is very soft along the critical line when the color superconductivity is incorporated, which may imply that there are large fluctuations of various observables including diquark-density mixed ones.

T.K. is grateful to Krishna Rajagopal for his valuable comments. This work was partially supported by a Grant-in-Aid for Scientific Research by the Ministry of Education, Culture, Sports, Science and Technology (MEXT) of Japan (Nos. 20540265, 19.07797), by Yukawa International Program for Quark-Hadron Sciences, and by the Grant-in-Aid for the global COE program " The Next Generation of Physics, Spun from Universality and Emergence " from MEXT.

\section{References}

[1] M. Asakawa and K. Yazaki, Chiral restoration at finite density and temperature, Nucl. Phys. A 504 (1989) 668; A. Barducci, R. Casalbuoni, S. De Curtis, R. Gatto and G. Pettini, Chiral symmetry breaking in QCD at finite temperature and density, Phys. Lett. B 231 (1989) 463; Phys. Rev. D 41 (1994) 1610.

[2] S. Ejiri, On the existence of the critical point in finite density lattice QCD, Phys. Rev. D 77 (2008), 014508; Canonical partition function and finite density phase transition in lattice $Q C D$, Phys. Rev. D 78 (2008) 074507.

[3] M. Kitazawa, T. Koide, T. Kunihiro and Y. Nemoto, Chiral and color superconducting phase transitions with vector interaction in a simple model, Prog. Theor. Phys. 108 (2002) 929; Z. Zhang, K. Fukushima and T. Kunihiro, Number of the QCD critical points with neutral color superconductivity, Phys. Rev. D 79 (2009) 014004; Z. Zhang and T. Kunihiro, Vector interaction, charge neutrality and multiple chiral critical point structures, Phys. Rev. D 80 (2009) 014015.

[4] T. Hatsuda, M. Tachibana, N. Yamamoto and G. Baym, New critical point induced by the axial anomaly in dense QCD, Phys. Rev. Lett. 97 (2006) 122001.

[5] P. de Forcrand and O. Philipsen, The chiral critical line of $N_{f}=2+1 Q C D$ at zero and non-zero baryon density, JHEP 0701 (2007) 077; K. Fukushima, Critical surface in hot and dense QCD with the vector interaction, Phys. Rev. D 78 (2008) 114019.

[6] P. C. Hohenberg and B. I. Halperin, Theory of Dynamic Critical Phenomena, Rev. Mod. Phys. 49 (1977) 435 .

[7] H.Fujii,Scalar density fluctuation at critical end point in NJL model, Phys. Rev .D 67 (2003) 094018; H.Fujii and M.Ohtani, Sigma and hydrodynamic modes along the critical line, Phys. Rev. D 70 (2004) 014016. 
[8] D.T.Son and M.A.Stephanov, Dynamic universality class of the QCD critical point, Phys. Rev. D 70 (2004) 056001.

[9] T. Kunihiro, Quark number susceptibility and fluctuations in the vector channel at high temperatures, Phys. Lett. B 271 (1991) 395.

[10] T. Kunihiro, Chiral transition and baryon-number susceptibility, CONFINEMENT 2000, Edited by H. Suganuma, M. Fukushima, H. Toki, ( Singapore, World Scientific, 2001) [arXiv:hep-ph/0007173].

[11] Y. Hatta and T. Ikeda, Universality, the QCD critical / tricritical point and the quark number susceptibility, Phys. Rev. D 67 (2003) 014028.

[12] G. D. Moore and O. Saremi, Bulk viscosity and spectral functions in QCD, JHEP 0809 (2008) 015.

[13] A. Onuki, Dynamic equations and bulk viscosity near the gas-liquid critical point, Phys. Rev. E 55 (1997) 403.

[14] Y. Minami and T. Kunihiro, Dynamical Density Fluctuations around QCD Critical Point Based on Dissipative Relativistic Fluid Dynamics - possible fate of Mach cone at the critical point—, Prog. Theor. Phys. 122 (2009) 881; [arXiv: 0904.2270 [hep-th] ].

[15] H. E. Stanley, Introduction to Phase Transitions and Critical Phenomena,(Oxford University Press, Oxford, 1977).

[16] As a latest reference, see B. I. Abelev et al. [STAR Collaboration], Indications of Conical Emission of Charged Hadrons at RHIC, Phys. Rev. Lett. 102 (2009) 052302.

[17] H. Stoecker, Collective Flow signals the Quark Gluon Plasma, Nucl. Phys. A 750 (2005) 121; L. M. Satarov, H. Stoecker and I. N. Mishustin, Mach shocks induced by partonic jets in expanding quark-gluon plasma, Phys. Lett. B 627 (2005) 64; J. Casalderrey-Solana, E. V. Shuryak and D. Teaney, Conical flow induced by quenched QCD jets, J. Phys. Conf. Ser. 27 (2005) 22 [Nucl. Phys. A 774 (2006) 577].

[18] L. D. Landau and E. M. Lifshitz, Fluid Mechanics (Pergamon,New York, 1959).

[19] C. Eckart, The Thermodynamics of irreversible processes. 3. Relativistic theory of the simple fluid, Phys. Rev. 58 (1940) 919.

[20] W. A. Hiscock and L. Lindblom, Generic instabilities in first-order dissipative relativistic fluid theories, Phys. Rev. D 31 (1985) 725.

[21] W. Israel, Nonstationary Irreversible Thermodynamics: A Causal Relativistic Theory, Ann.Phys.(N.Y.) 100 (1976) 310; W. Israel and J.M.Stewart, Transient relativistic thermodynamics and kinetic theory, Ann. Phys.(N.Y.) 118(1979) 341.

[22] K. Tsumura, T. Kunihiro and K. Ohnishi, Derivation of covariant dissipative fluid dynamics in the renormalization-group method, Phys. Lett. B 646 (2007) 134.

[23] K. Tsumura, T. Kunihiro, Stable First-order Particle-frame Relativistic Hydrodynamics for Dissipative Systems , Phys. Lett. B 668 (2008) 425.

[24] L. P. Kadanoff and P. C. Martin, Hydrodynamic Equations and Correlation Functions, Ann. Phys. 24 (1963) 419; R. D. Mountain, Spectral Distribution of Scattered Light in a Simple Fluid, Rev. Mod. Phys. 38(1966) 205.

[25] L. E. Reichl, A Modern Course in Statistical Physics(Wiley-Interscience 1998).

[26] K. Tsumura, Y. Minami and T. Kunihiro, in progress. 